\title{
COUNTABLE MODELS OF STABLE THEORIES
}

\author{
ANAND PILIAY ${ }^{1}$
}

\begin{abstract}
The notion of a normal theory is introduced, and it is proved that for such a theory $T, I\left(\boldsymbol{\kappa}_{0}, T\right)=1$ or $\geqslant \boldsymbol{\kappa}_{0}$. We also include a short proof of Lachlan's theorem that for superstable $T, I\left(\boldsymbol{\aleph}_{0}, T\right)=1$ or $\geqslant \boldsymbol{N}_{0}$. (The property of normality is stronger than stability but incomparable to superstability.)
\end{abstract}

1. Preliminaries. We consider here theories $T$ which are countable and complete (and of course finitary, first order). A long standing question is whether for stable. not $\boldsymbol{\aleph}_{0}$-categorical $T, T$ must always have infinitely many countable models (up to isomorphism). This was answered positively by Lachlan [1] for $T$ superstable, and other proofs in this case have been given by Lascar [3] and Shelah [7]. Here we consider theories which satisfy, in effect, a stronger combinatorial property than "not having the order property", and answer the question positively for such theories, strengthening results in Pillay [6]. I wish to thank the referee for his suggestions, especially concerning the proof of Lemma 4 and an error in our earlier formulation of Lemma 6.

Definition 1. (i) The $L$-formula $\alpha(\bar{x}, \bar{y})$ is $T$-normal (or just normal) if for any $\bar{a}, \bar{b}$ in a model $M$ of $T$ the definable subsets $\alpha(\bar{x}, \bar{a})$ and $\alpha(\bar{x}, \bar{b})$ are either disjoint or equal.

(ii) $T$ is normal if every $L$-formula is equivalent modulo $T$ to a Boolean combination of normal formulae (i.e. formulae that are normal no matter how we separate the variables). (Our use of the word normal is motivated by the normal formulas of Lachlan [2], but our notion is of course much cruder.)

It is easy to see that a normal theory is stable, either by counting types (if $\alpha(x, \bar{y})$ is normal then there can be at most $\lambda \alpha$-types over any model of power $\lambda$ ) or by observing that a normal formula does not have the order property and thus neither does any Boolean combination of such formulae. Clearly, any theory of equivalence relations $E_{i}$ which has elimination of quantifiers is normal, and thus there are normal theories which are $\omega$-stable, strictly superstable and strictly stable. Any theory of $R$-modules is normal, as a consequence of Baur's "p.p elimination of quantifiers" for modules, and the fact that in an $R$-module $M$, if $\varphi(\bar{x}, \bar{y})$ is a p.p (positive primitive) formula, then for any $\bar{a} \in M \varphi(\bar{x}, \bar{a})$ defines (if consistent) a coset of the subgroup

Received by the editors November 30. 1982. Part of the contents of this paper was presented at the 797 th meeting of the A. M. S. at the University of Maryland, October 1982.

1980 Mathematics Subject Classification. Primary 03C45: Secondary 03C15.

Key nords and phrases. Normal theory. superstable.

'Supported by a grant from the NSERC of Canada. 
$\varphi(\bar{x}, \bar{o})$ of $M^{\prime \prime}$. Finally, it is an easy exercise to show that any theory of one equivalence relation is normal (although such a theory must also be $\omega$-stable). Thus, there are normal theories which have the f.c.p. Our proof (in §3) that for $T$ normal, $I\left(\boldsymbol{\aleph}_{0}, T\right)$ is one or infinite, does not actually use the machinery of stability theory, but uses directly the combinatorial properties of Boolean combinations of normal formulae.

Our proof (in §2) of Lachlan’s theorem uses just the basic properties of forking (which can be found in [4 or 7]), and does not use, for example, the notion of weight. In fact, our proof is in a sense just an updated version of Lachlan's original proof, although more information is obtained. Another proof of this theorem is contained in Mustafin [5].

Both our proofs involve a notion which we call serini-isolation, and which seems quite useful. We say that $\bar{a}$ semi-isolates $\bar{b}$ if there is a formula $\varphi(\bar{a}, \bar{x})$ satisfied by $\bar{b}$, which also determines the pure type of $\bar{b}$. This relation is transitive. Moreover, if $T$ is stable, and we restrict ourselves to tuples realising nonisolated types, then semiisolation implies forking. Thus we have a transitive relation which implies forking, which is quite a strong property. For the sake of completeness we give the definition of semi-isolation in more generality.

Definition 2. Let $A$ be a set and $\bar{a}, \bar{b}$ tuples. We say that $\bar{a}$ semi-isolates $\bar{b}$ over $A$ if there is a formula $\psi(\bar{x}) \in \operatorname{tp}(\bar{b} / A \cup \bar{a})$ such that $\psi(\bar{x}) \vDash \operatorname{tp}(\bar{b} / A)$. If $A=\varnothing$ we omit "over $A$ ". We also say that such $\psi(\bar{x})$ as above witnesses the fact that $\bar{a}$ semi-isolates $\bar{b}$ over $A$.

OBSERVATION 3. The relation of semi-isolation over $A$ is transitive; namely, if $\bar{a}$ semi-isolates $\bar{b}$ over $A$ and $\bar{b}$ semi-isolates $\bar{c}$ over $A$, then $\bar{a}$ semi-isolates $\bar{c}$ over $A$.

Proof. We observe that if $\psi_{1}(\bar{a}, \bar{x}) \in L(A)$ witnesses $\bar{a}$ semi-isolating $\bar{b}$ over $A$ and $\psi_{2}(\bar{b}, \bar{y}) \in L(A)$ witnesses $\bar{b}$ semi-isolating $\bar{c}$ over $A$, then the formula $(\exists \bar{x})\left(\psi_{1}(\bar{a}, \bar{x}) \wedge \psi_{2}(\bar{x}, \bar{y})\right)$ witnesses $\bar{a}$ semi-isolating $\bar{c}$ over $A$.

Lemma 4. Let $T$ be a stable. Let $A, \bar{a}$ and $\bar{b}$ be such that $\bar{a}$ semi-isolates $\bar{b}$ over $A$, but $\operatorname{tp}(\bar{b} / A)$ is not isolated (i.e. nonprincipal). Then $\operatorname{tp}(\bar{b} / A \cup \bar{a})$ forks over $A$.

Proof. This follows directly from the "Open Map Theorem" [4]. However, we will present a "simpler" proof. First, suppose $A$ to be countable. Let $\psi(\bar{a}, \bar{y}) \in L(A)$ witness $\bar{a}$ semi-isolating $\bar{b}$ over $A$. Let $p(\bar{y})=\operatorname{tp}(\bar{b} / A)$. So $\vDash \psi(\bar{a}, \bar{y}) \rightarrow p(\bar{y})$. As $p$ is nonisolated, there is (by the Omitting Types theorem and countability of $A$ ) a model $M \supset A$ such that $p$ is not realised in $M$. It follows that for no $\bar{b}^{\prime} \in M$ do we have $\vDash \psi\left(\bar{a}, \bar{b}^{\prime}\right)$. Thus, $\psi(\bar{x}, \bar{y})$ is not in the bound of $\operatorname{tp}(\bar{a} / A)$, but is represented in $\operatorname{tp}(\bar{a} / A \cup \bar{b})$, whereby $\operatorname{tp}(\bar{a} / A \cup \bar{b})$ forks over $A$. By forking symmetry, $\operatorname{tp}(\bar{b} / A \cup \bar{a})$ forks over $A$.

The general case is reduced to the case $A$ countable by the following: Let $\bar{a}, \bar{b}, A$ be such that $\bar{a}$ semi-isolates $\bar{b}$ over $A, \operatorname{tp}(\bar{b} / A)$ is not isolated and $\operatorname{tp}(\bar{b} / A \cup \bar{a})$ does not fork over $A$. Then there is countable $A_{0} \subset A$ such that the previous sentence holds with $A$ replaced by $A_{0}$. 
2. The case $T$ superstable. We know that a theory $T$ is superstable if and only if (i) $T$ is stable, and (ii) there do not exist types $p_{i}(\bar{x}) \in S\left(A_{i}\right)$ for $i<\omega$ such that $A_{i} \subset A_{i+1}, p_{i} \subset p_{i+1}$ for $i<\omega$ and $p_{i+1}$ is a forking extension of $p_{i}$.

((ii) just says that $\kappa(T)=\boldsymbol{\aleph}_{0}$.)

A simple consequence of this, together with forking symmetry, is

FACT 5. Let $T$ be superstable. Then there do not exist tuples $\bar{a}$ and $\bar{b}_{i}$ for $i<\omega$ such that $\left\{\bar{b}_{i}: i<\omega\right\}$ is independent, but for each $i<\omega \operatorname{tp}\left(\bar{b}_{i} / \bar{a}\right)$ forks over $\varnothing$.

(Let us note that for $T$ stable we can define a type $p \in S(A)$ to be superstable if (ii) above holds with $p_{0}=p$ (i.e. $R(p)<\infty$ for a suitable rank $R$ ). In that case, Fact 5 holds under the new assumptions that $T$ is stable and $\operatorname{tp}(\bar{a})$ is superstable.)

Everything is contained in the following lemma.

Lemma 6. Let $T$ be superstable. Then there do not exist $\bar{a}_{0}, \bar{a}, \bar{b}$ such that $\operatorname{tp}\left(\bar{a}_{0}\right)=\operatorname{tp}(\bar{a}), \operatorname{tp}(\bar{b})$ is nonisolated, $\operatorname{tp}\left(\bar{a}^{\wedge} \bar{b} / \bar{a}_{0}\right)$ is isolated and $\{\bar{a}, \bar{b}\}$ is independent (over $\varnothing$ ).

Proof. Let us assume that there exist such $\bar{a}_{0}, \bar{a}, \bar{b}$ and arrive at a contradiction. Clearly, $\bar{a}_{0}$ semi-isolates $\bar{a}$ and $\bar{a}_{0}$ semi-isolates $\bar{b}$. Let us define $\bar{a}_{i}, \bar{b}_{i}$ for $1 \leqslant i<\omega$ as follows: $\bar{a}_{1}=\bar{a}, \bar{b}_{1}=\bar{b}$. Suppose we have defined $\bar{a}_{i}, \bar{b}_{i}$ for $i \leqslant n$; then we choose $\bar{a}_{n+1}, \bar{b}_{n+1}$ such that $\operatorname{tp}\left(\bar{a}_{n} \backslash \bar{a}_{n+1} \wedge \bar{b}_{n+1}\right)=\operatorname{tp}\left(\bar{a}_{0} \wedge \bar{a}_{1} \wedge \bar{b}_{1}\right)$ and, moreover, $\operatorname{tp}\left(\bar{a}_{n+1} \wedge \bar{b}_{n+1} / \bar{a}_{0} \cup \cup_{1 \leqslant i \leqslant n} \bar{a}_{i} \wedge \bar{b}_{i}\right)$ does not fork over $\bar{a}_{n}$. This can clearly be obtained, and $\bar{a}_{i}$ semi-isolates both $\bar{a}_{i+1}$ and $\bar{b}_{i+1}$.

By induction and Observation 3, we have that $\bar{a}_{0}$ semi-isolates $\bar{a}_{i}$ for all $i<\omega$. Thus, again by Observation 3, we have:

(I) for each $i<\omega, \bar{a}_{0}$ semi-isolates $\bar{b}_{i}$.

I now claim

(II) for each $n<\omega\left\{\bar{b}_{1}, \bar{b}_{2}, \ldots, \bar{b}_{n}, \bar{b}_{n+1}, \bar{a}_{n+1}\right\}$ is independent over $\varnothing$.

We prove this claim by induction, the case $n=0$ being that $\{\bar{a}, \bar{b}\}$ is independent, which we are assuming. Fix $n$. By construction, $\operatorname{tp}\left(\bar{a}_{n+1} \wedge \bar{b}_{n+1} /\left\{\bar{b}_{1}, \ldots, \bar{b}_{n}, \bar{a}_{n}\right\}\right)$ does not fork over $\bar{a}_{n}$. Thus by forking symmetry, $\operatorname{tp}\left(\bar{b}_{1} \cdots \ldots \wedge \bar{b}_{n} /\left\{\bar{a}_{n+1}, \bar{b}_{n+1}, \bar{a}_{n}\right\}\right)$ does not fork over $\bar{a}_{n}$. But by the induction hypothesis, $\operatorname{tp}\left(\bar{b}_{1} \wedge \ldots \bar{b}_{n} / \bar{a}_{n}\right)$ does not fork over $\varnothing$. Thus, $\operatorname{tp}\left(\bar{b}_{1} \wedge \ldots \wedge \bar{b}_{n} /\left\{\bar{a}_{n+1}, \bar{b}_{n+1}\right\}\right)$ does not fork over $\varnothing$ (transitivity). Now, using the facts that $\left\{\bar{a}_{n+1}, \bar{b}_{n+1}\right\}$ is independent over $\varnothing$ $\left(\operatorname{tp}\left(\bar{a}_{n+1} \bar{b}_{n+1}\right)=\operatorname{tp}\left(\bar{a}^{\wedge} \bar{b}\right)\right)$ and $\left\{\bar{b}_{1}, \ldots, \bar{b}_{n}\right\}$ is independent over $\varnothing$ (induction hypothesis), it follows that $\left\{\bar{b}_{1}, \ldots, \bar{b}_{n}, \bar{b}_{n+1}, \bar{a}_{n+1}\right\}$ is independent over $\varnothing$. So (II) is established.

It follows from (II) that

(III) $\left\{\bar{b}_{i}: 1 \leqslant i<\omega\right\}$ is independent over $\varnothing$.

Now (I), Lemma 4 and the fact that $\operatorname{tp}(\bar{b})$ is nonisolated imply that for each $i$, $\operatorname{tp}\left(\bar{b}_{i} / \bar{a}_{0}\right)$ forks over $\varnothing$. This together with (III) contradicts superstability (i.e. contradicts Fact 5), and so proves the lemma.

Note. All we used in Lemma 6 was stability and the fact that $\operatorname{tp}\left(\bar{a}_{0}\right)$ was superstable. 
COROllary 7. Let $T$ be superstable, $p(x) \in S(T)$ and $M_{p}$ a model prime over a realisation of $p$. Then $M_{p}$ omits any type $q(\bar{x}, \bar{y})$ such that $q(\bar{x}, \bar{y}) \supset p(\bar{x})$ and $q(\bar{x}, \bar{y})$ "says" $\bar{x}$ and $\bar{y}$ are independent and $\operatorname{tp}(\bar{y})$ is nonisolated.

COROllary 8. Let $T$ be superstable, and $p \in S(T)$ a nonisolated type. Let $\left\{\bar{a}_{0}, \bar{a}_{1}, \bar{a}_{2}, \ldots\right\}$ be an independent set of realisations of $p$, and for each $n<\omega$ suppose that $M_{n}$ is prime over $\left\{\bar{a}_{0}, \bar{a}_{1}, \ldots, \bar{a}_{n}\right\}$. Then $M_{n} \not M_{m}$ for $n<m$.

Proof. By Corollary $7, M_{n}$ omits $\operatorname{tp}\left(\bar{a}_{0} \quad \bar{a}_{1} \quad \cdots \quad \bar{a}_{n} \bar{a}_{n+1}\right)$.

THEOREM 9. Let $T$ be superstable. Then $I\left(\boldsymbol{\aleph}_{0}, T\right)=1$ or $I\left(\boldsymbol{\aleph}_{0}, T\right) \geqslant \boldsymbol{\aleph}_{0}$.

Proof. Assume $I\left(\boldsymbol{\aleph}_{0}, T\right) \neq 1$. So there is some nonisolated type $p(\bar{x}) \in S(T)$. We may assume that $S(T)$ is countable, and thus there are prime models over any finite set. The theorem now follows from Corollary 8.

Let me finally note that the proofs by Lascar and Shelah of Theorem 9 (which actually give less information than is contained in say Corollary 8) use the deep fact that for $T$ superstable and $\bar{a}$ a given tuple, we can put a finite bound on the size of a set $I$ for which there can be a set $\left\{\bar{b}_{i}: i \in I\right\}$ which is independent, and for which $\operatorname{tp}\left(\bar{b}_{i} / \bar{a}\right)$ forks over $\varnothing$ for all $i \in I$. The least such bound is called the weight of $\operatorname{tp}(\bar{a})$.

3. The case $T$ normal. The basic idea here is to show that if $T$ is normal and $S(T)$ countable, then for "enough" types $p \in S(T)$ the relation of semi-isolation restricted to realisations of $p$ is an equivalence relation. We do not even know if this is true for an arbitrary superstable theory, so the proof here is by no means a generalisation of the proof in $\$ 2$.

Definition 10. Let $p \in S(T)$. We denote by $S_{p}$ the relation of semi-isolation (over $\varnothing$ ) restricted to realisations of $p$.

LEMma 11. Let $p \in S(T)$ be nonisolated and suppose that $S_{p}$ is an equivalence relation. Suppose that $M_{p}$ is a model prime over a realisation of $p$. Then $M_{p}$ omits some type $q \in S(T)$.

Proof. Let $\bar{a}$ realize $p$ in $M_{p}$ such that $M_{p}$ is prime over $\bar{a}$. So for all $\bar{b}$ realising $p$ in $M_{p}, \bar{a}$ semi-isolates $\bar{b}$ (in fact $\operatorname{tp}(\bar{b} / \bar{a})$ is isolated). Thus $S_{p}$ has only one equivalence class in $M_{p}$. On the other hand, it is easy, as $p$ is nonisolated, to find a type $q(\bar{x}, \bar{y}) \in S(T)$ such that $p(\bar{x}) \cup p(\bar{y}) \subset q(\bar{x}, \bar{y})$ and for all realisations $\bar{a}^{\prime \prime} \bar{b}^{\prime}$ of $q, \bar{a}^{\prime}$ does not semi-isolate $\bar{b}^{\prime}$. So $q$ is omitted in $M_{p}$, proving the lemma.

I assume familiarity with the Cantor-Bendixon (CB) rank on types and formulae. All one has to know are the following facts: $S_{n}(T)$ is countable iff for all $p \in S_{n}(T)$, $\mathrm{CB}_{n}(p)<\infty$. For $\varphi$ an $n$-formula $\mathrm{CB}_{n}(\varphi)$ is defined to be $\sup \left\{\mathrm{CB}_{n}(p): p \in\right.$ $\left.S_{n}(T), \varphi \in p\right\}$. Moreover, if $p \in S_{n}(T)$ and $\mathrm{CB}_{n}(p)=\alpha<\infty$, then there is $\varphi \in p$ such that $\mathrm{CB}_{n}(\varphi)=\alpha$ and $p$ is the unique $n$-type of $\mathrm{CB}_{n}$ rank $\geqslant \alpha$ containing $\varphi$. For $p \in S_{n}(T), \mathrm{CB}(p)$ means $\mathrm{CB}_{n}(p)$.

I will now state without proof a crucial proposition, state and prove the main theorem, and then set about proving the proposition. 
Proposition 12. Let $T$ be normal, and $K$ a nonempty class of models of $T$. Let $p \in S(T)$ be such that $\mathrm{CB}(p)<\infty, p$ is omitted in all $M \in K$ and, moreover, $p$ is of least $\mathrm{CB}$ rank among the types omitted in all $M \in K$. Then $S_{p}$ is an equivalence realtion.

TheOREM 13. Let $T$ he normal. Then $I\left(\boldsymbol{\aleph}_{0}, T\right)=1$ or $I\left(\boldsymbol{\aleph}_{0}, T\right) \geqslant \boldsymbol{\aleph}_{0}$.

Proof. Assume that $I\left(\boldsymbol{\aleph}_{0}, T\right) \neq 1$. We may also assume that $S(T)$ is countable (otherwise clearly $T$ has uncountably many, in fact $2^{\aleph_{0}}$; countable models). Thus there are prime models over all finite sets, and all types have $\mathrm{CB}$ rank $<\infty$. Let $K=\{M: M \vDash T, M$ countable and $M$ omits some type in $S(T)\} . K$ is nonempty, as there is a prime model, which omits any nonisolated type. If $K$ contains infinitely many isomorphism types of models, then we are finished. Otherwise, the isomorphism types in $K$ are $\left\{M_{1}, M_{2}, \ldots, M_{n}\right\}$. Each $M_{n}$ omits some $p_{n} \in S(T)$. Thus, there is clearly some $p \in S(T)$ which is omitted in all the $M_{i}$. Choose such a $p$ of least possible CB rank, and let $M_{p}$ be prime over a realisation of $p . M_{p}$ is countable and by Lemma 11 and Proposition 12, $M_{p}$ omits some $q \in S(T)$. But then $M_{p} \in K$, which clearly gives a contradiction. So the theorem is proved.

Now we proceed to prove Proposition 12. We need the following combinatorial fact about Boolean combinations of normal formulae.

Lemma 14. Let $\alpha(\bar{x}, \bar{y})$ be $\varphi(\bar{x}, \bar{y}) \wedge \wedge_{r=1}^{k} \neg \psi_{r}(\bar{x}, \bar{y})$ where $\varphi$ and the $\psi_{r}$ are normal. Let $m<\omega$ be such that $m \rightarrow(3)_{k}^{2}$. Then there do not exist $\bar{a}_{i}, \bar{b}_{i}$ for $i=1, \ldots, m$, and $\bar{b}$ such that:

(i) $\vDash \alpha\left(\bar{a}_{i}, \bar{b}_{i}\right) \wedge \alpha\left(\bar{a}_{i}, \bar{b}\right)$ for $1 \leqslant i \leqslant m$, and

(ii) $\vDash \neg \alpha\left(\bar{a}_{j}, \bar{b}_{i}\right)$ for all $1 \leqslant i<j \leqslant m$.

Proof. First note that $m$, as in the lemma, exists by the finite version of Ramsey's theorem. Now suppose by way of contradiction that there exist $\bar{a}_{i}, \bar{b}_{i}$ for $1 \leqslant i \leqslant m$ and $\bar{b}$ satisfying (i) and (ii). Then first of all, we must have

(I) $\vDash \varphi\left(\bar{a}_{i}, \bar{b}_{j}\right)$ for all $i, j$ with $1 \leqslant i, j \leqslant m$. For, pick any such $i, j$. We have $\vDash \varphi\left(\bar{a}_{i}, \bar{b}\right) \wedge \varphi\left(\bar{a}_{j}, \bar{b}\right) \wedge \varphi\left(\bar{a}_{j}, \bar{b}_{j}\right)$ from which $\vDash \varphi\left(\bar{a}_{i}, \bar{b}_{j}\right)$ follows by normality of $\varphi$.

An immediate consequence of (I) and the assumption that $\vDash \neg \alpha\left(\bar{a}_{j}, \bar{b}_{i}\right)$ whenever $1 \leqslant i<j \leqslant m$ is

(II) $\vDash \vee_{r=1}^{k} \psi_{r}\left(\bar{a}_{j}, \bar{b}_{i}\right)$ whenever $1 \leqslant i<j \leqslant m$. Let $[m]^{2}$ denote the set of unordered pairs $\{i, j\}$ with $1 \leqslant i, j \leqslant m$, and define the function $f:[m]^{2} \rightarrow\{1, \ldots, k\}$. If $1 \leqslant i<j \leqslant m$, then $f(i, j)=$ the least $r<k$ such that $\vDash \psi_{r}\left(\bar{a}_{j}, \bar{b}_{i}\right)$ (which is well defined by (II)). By the assumption on $m$, there is $X \subseteq\{1, \ldots, m\}$ with $|X|=3$, and there is $r_{1} \leqslant k$ such that $f(i, j)=r_{1}$ for all $i<j$ in $X$. Let $X=\left\{i_{1}, i_{2}, i_{3}\right\}$ with $i_{1}<i_{2}<i_{3}$. So

$$
\vDash \psi_{r_{1}}\left(\bar{a}_{i_{3}}, \bar{b}_{i_{1}}\right) \wedge \psi_{r_{1}}\left(\bar{a}_{i_{3}}, \bar{b}_{i_{2}}\right) \wedge \psi_{r_{1}}\left(\bar{a}_{i_{2}}, \bar{b}_{i_{1}}\right) \text {. }
$$

As $\psi_{r_{1}}$ is normal we obtain $\vDash \psi_{r_{1}}\left(\bar{a}_{i_{2}}, \bar{b}_{i_{2}}\right)$, which contradicts the assumption that $\vDash \alpha\left(\bar{a}_{i_{2}}, \bar{b}_{i_{2}}\right)$. So the lemma is proved. 
Proof of Proposition 12. Let $T$ be normal and $K, p$ be as in the statement of the proposition. Let $\gamma<\infty$ be such that $\mathrm{CB}(p)=\gamma$. Let $\varphi(\bar{x}) \in p$ be such that $\mathrm{CB}(\varphi)=\gamma$ and for all $q$, if $\mathrm{CB}(q) \geqslant \gamma$ and $\varphi \in q$ then $q=p$ ( $\varphi$ isolates $p$ among types of $\mathrm{CB}$ rank $\geqslant \gamma$ ).

Let $\bar{a}, \bar{b}$ be realisations of $p$ such that $\bar{a}$ semi-isolates $\bar{b}$. We must show that $\bar{b}$ semi-isolates $\bar{a}$. (We already know $S_{p}$ to be transitive and it is clearly reflexive). As $\bar{a}$ semi-isolates $\bar{b}$ there is an $L$-formula $\alpha(\bar{x}, \bar{y})$ such that $\vDash \alpha(\bar{a}, \bar{b})$ and $k \alpha(\bar{a}, \bar{y}) \rightarrow$ $p(\bar{y})$. As $T$ is normal, we can take $\alpha$ to be a disjunction of conjunctions of normal and negated normal formulae. By choosing a suitable disjunct and noticing that a conjunction of normal formulae is normal, we may assume that $\alpha(\bar{x}, \bar{y})$ is the conjunction of one normal and a finite set of negated normals. (So $\alpha(\bar{x}, \bar{y})$ satisfies the assumption of Lemma 14 , for suitable $m$.)

Let us now suppose that $\bar{b}$ does not semi-isolate $\bar{a}$ and we will obtain a contradiction.

I shall define inductively $\bar{a}_{i}, \bar{b}_{i}$ (in the universe) for $i<\omega$ such that:

(i) $\vDash \alpha\left(\bar{a}_{i}, \bar{b}_{i}\right) \wedge \alpha\left(\bar{a}_{i}, \bar{b}\right)$ for all $i<\omega$,

(ii) $\vDash \neg \alpha\left(\bar{a}_{j}, \bar{b}_{i}\right)$ whenever $i<j<\omega$, and,

(iii) $\mathrm{CB}\left(\operatorname{tp}\left(b_{i}\right)\right)<\gamma$ for all $i<\omega$.

Suppose that we have already defined $\bar{a}_{i}$ and $\bar{b}_{i}$ for $i<n$ satisfying the required conditions. We proceed to define $\bar{a}_{n}$ and $\bar{b}_{n}$.

Let $\psi_{i}(\bar{x})$ for $i<n$ be $L$-formulae such that $\vDash \psi_{i}\left(\bar{b}_{i}\right)$ for each $i<n$ and $\operatorname{CB}\left(\psi_{i}(\bar{x})\right)$ $<\gamma$ for each $i<n$ (by induction hypothesis (iii)).

As $\vDash \alpha(\bar{a}, \bar{y}) \rightarrow p(\bar{y})$ and $\mathrm{CB}(p)=\gamma$ it follows that

$$
\vDash \alpha(\bar{a}, \bar{y}) \rightarrow \underset{i<n}{\wedge} \neg \psi_{i}(\bar{y})
$$

Let $\psi(\bar{x})$ be the formula $(\forall \bar{y})\left(\alpha(\bar{x}, \bar{y}) \rightarrow \bigwedge_{i<n} \neg \psi_{i}(\bar{y})\right)$, and let $\beta(\bar{x}, \bar{y})$ denote the $L$-formula $\varphi(\bar{x}) \wedge \psi(\bar{x}) \wedge \alpha(\bar{x}, \bar{y}) \wedge \varphi(\bar{y})$. It is then clear from our assumptions, and $(*)$ that $\vDash \beta(\bar{a}, \bar{b})$.

As $\bar{b}$ does not semi-isolate $\bar{a}$, there is an $\bar{a}^{\prime}$ somewhere such that

$$
\vDash \beta\left(\bar{a}^{\prime}, \bar{b}\right) \text { and } \operatorname{tp}\left(\bar{a}^{\prime}\right) \neq p .
$$

However, $\bar{a}^{\prime}$ satisfies $\varphi(\bar{x})$, so by choice of $\varphi(\bar{x})$ we must have that $\operatorname{CB}\left(\operatorname{tp}\left(\bar{a}^{\prime}\right)\right)<\gamma$. As $p$ was chosen to be of least $\mathrm{CB}$ rank omitted in all $M \in K$, it follows that $\operatorname{tp}\left(\bar{a}^{\prime}\right)$ is realized in some $M \in K$, and so w.l.o.g. $\bar{a}^{\prime} \in M$ (some $M \in K$ ).

So $M \vDash(\exists \bar{y}) \beta\left(\bar{a}^{\prime}, \bar{y}\right)$ (as $M$ is an elementary submodel of the universe).

$$
\text { Let } \overline{b^{\prime}} \in M \text { be such that } M \vDash \beta\left(\bar{a}^{\prime}, \overline{b^{\prime}}\right) \text {. }
$$

We will put $\bar{a}^{\prime}=\bar{a}_{n}$ and $\bar{b}^{\prime}=\bar{b}_{n}$, and by $(* *)$ and $(* * *)$ condition (i) is satisfied.

Clearly, $\bar{b}_{n}$ satisfies $\varphi(\bar{x})$, but as $M \in K, p$ is omitted in $M$, whereby (by choice of 甲) $\mathrm{CB}\left(\operatorname{tp}\left(\bar{b}_{n}\right)\right)<\gamma$, satisfying condition (iii).

So we only have to check that condition (ii) is satisfied; namely that $\vDash \neg \alpha\left(\bar{a}_{n}, \bar{b}_{i}\right)$, for all $i<n$. 
But this follows from the fact that $\bar{a}_{n}$ satisfies $\psi(\bar{x})$; namely that

$$
\vDash(\forall \bar{y})\left(\alpha\left(\bar{a}_{n}, \bar{y}\right) \rightarrow \bigwedge_{i<n} \neg \psi_{i}(\bar{y})\right)
$$

and that $\bar{b}_{i}$ satisfies $\psi_{i}$ for each $i<n$.

Thus, the $\bar{a}_{i}, \bar{b}_{i}$ an be defined as required. But this contradicts Lemma 14. Thus Proposition 12 is proved.

\section{REFERENCES}

1. A. H. Lachlan, The number of countable models of a countable superstable theorr. Proc. Internat Congr. on Logic. Methodology and the Philosophy of Science, North-Holland, Amsterdam, 1973, pp. $45-56$.

2. Tho conjectures on the stability of $\omega$-categorial theories, Fund. Math. 81 (1974), 133-145.

3. D. Lascar, Ranks and definability in superstable theories, Israel J. Math. 23 (1976), 53-87.

4. D. Lascar and B. Poizat, An introduction to forking, J. Symbolic Logic 44 (1979), 330-350.

5. T. (j. Mustafin. Number of countable models of a countable complete theory. Algebra i Logika 20 (1981), 69-91.

6. A. Pillay. Countable modules, Fund. Math. (to appear).

7. S. Shelah, Classification theory and the number of non-isomorphic models, North-Holland, Amsterdam. 1978.

Department of Mathematics, McGill University, Montreal, Quebec, Canada H3A 2K6

Current address: Department of Mathematics, University of Notre Dame, Notre Dame, Indiana 46556 